\title{
SEM - based construction cost analysis of prefabricated building
}

\author{
Hongxiong Yang ${ }^{1}$, Yunpeng Wang ${ }^{2 * *}$ \\ ${ }^{1}$ School of Management, Tianjin University of Technology, Tianjin China. \\ ${ }^{2}$ School of Management, Tianjin University of Technology, Tianjin China.
}

\begin{abstract}
Prefabricated buildings have comprehensive advantages over traditional buildings, but the reasons for the incremental cost in the construction stage are complex and need to be solved urgently. This paper takes the prefabricated components that have the greatest impact on the construction cost of prefabricated buildings as the research object, divides the construction process of prefabricated components into three stages: production, transportation and site installation of prefabricated components, studies the interrelation of each stage in combination with the application of information technology system of prefabricated components, and establishes a model by using structural equation. The results show that the production cost of prefabricated parts has a positive influence on the transportation and installation cost. Transportation and the application of information technology have the greatest impact on construction costs; the production and assembly of prefabricated parts have relatively little influence on the construction cost.
\end{abstract}

\section{The introduction}

Prefabricated building can save materials, shorten the construction period and improve the construction environment quality, but its market share is not high. Studies have shown that the production cost of prefabricated parts has a great impact on prefabricated buildings ${ }^{[1,2]}$, but the multi-dimensional operation of prefabricated parts is not considered. Other scholars believe that the transportation cost of prefabricated parts accounts for a large proportion ${ }^{[3,4]}$, but they fail to consider other factors. Barlow et al. ${ }^{[5]}$ hold that information technology can reduce the construction cost through the application of information technology in residential construction. The existing research results do not systematically consider the coupling of each work space of prefabricated parts, and the conclusion is that it is difficult to effectively control the construction cost of prefabricated buildings.

Therefore, this paper takes the prefabricated parts as the research object, constructs the structural equation model, combines the application of information technology with the three different operation dimensions of the prefabricated parts, systematically considers the mutual coupling of each stage, finds out the key factors affecting the construction cost of prefabricated buildings, and puts forward corresponding countermeasures.

\section{Research scope definition and key factors selection}

\subsection{Scope Definition}

This paper takes the prefabricated parts as the research object and defines the construction cost in terms of the whole construction process of the prefabricated parts, which is a more generalized construction cost. From the perspective of general contracting of prefabricated building, this paper studies the construction cost of all prefabricated building projects rather than individual construction cost.

\subsection{Selection of key factors}

In the subject of CNKI retrieval, "Prefabricated construction cost", the factors influencing the construction cost are summarized according to the four aspects of prefabricated parts production, transportation, assembly cost and application of information technology. Among them, the measurement variables of the production cost of prefabricated parts (PR) are the degree of standardization of prefabricated parts (SD), labor cost (LC), material cost (MAC), fixed costs such as models (MEC), regular inspection of prefabricated parts (CI), storage and maintenance (SM). The measurement variables for the prefabricated part transport (TR) are vehicle scheduling (VS), transport vehicle selection (TM), prefabricated part site selection (SC), route selection and optimization (RO), loading and unloading and prefabricated part protection equipment (LPE), and prefabricated part transport rate (CTR). Measurement variables of on-site assembly of prefabricated parts (CA) are prefabricated part connection (PCC), vertical transport machinery (HM), professional operator (MO), and secondary transport (RT). The measurement variables of the application of information technology (IM) of prefabricated components are BIM technology application (BIM), cloud computing technology application (CM) and RFID technology application (RFID).

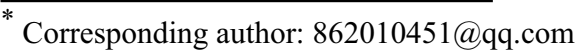




\section{Structural equation model and data analysis}

\subsection{Structural equation model}

Structural equation model (SEM) can simultaneously deal with multiple causes and relationship between results, and measure the overall fitting degree of the model. Considering that the factors affecting the cost have interactive relations, the structural equation model is selected as the research tool. The theoretical model is shown in Figure 1.

\section{Component transport \\ Component \\ production}

component
composition

\section{Components apply information technology}

The construction cost of prefabricated buildings
Fig.1. Factors influencing the construction cost of prefabricated buildings

Make assumptions about each path in the model:

H1: The higher the input cost of the application of information technology in prefabricated parts, the better it can control the transportation cost, production cost, on-site assembly cost and construction cost of prefabricated parts.

$\mathrm{H} 2$ : The more input into the production cost of prefabricated parts, the more effective it is to control the transportation cost and assembly cost.

H3: The higher the cost of transportation, production and assembly of prefabricated parts, the higher the construction cost.

\subsection{Data Sources}

Through field research and literature reading, the structural equation model was established. The model was investigated by questionnaire, and the influence degree of each factor was scored by Likert scale. "1" means no influence, "5" means great influence, and the influence degree from "1" to "5" increases step by step. Questionnaires were sent by mail to experts in prefab construction enterprises and researchers in universities and colleges. A total of 215 questionnaires were issued, 182 of which were effectively recovered, with an effective recovery rate of $85 \%$.

\subsection{Reliability and validity analysis}

SPSS 25.0 was used to analyze the reliability and validity of the survey data. The reliability coefficient Cronbach's was 0.832 , indicating a high reliability. KMO value is 0.858 , suitable for factor analysis, Bartlett sphere test is
0.000 , statistical significance, each indicator is strongly correlated. At the same time, four factors with characteristic roots greater than 1 were analyzed, which indicated that the study had good structural validity. The Cronbach's $\alpha$ reliability coefficients of the four potential variables were all greater than 0.6 , which were within the acceptable range.

\section{Structural equation model analysis}

\subsection{Structural equation model verification}

Taking the prefabricated parts as the object, AMOS 23.0 software was used to simulate and verify the relationship between the influencing factors of the construction cost of prefabricated buildings, and a standardized model of confirmatory factor analysis was obtained. All values in the model fitting are within acceptable range, which indicates that the fitting is better and the model establishment is scientific and reasonable.

According to the model, the standardization coefficient is concentrated between 0.52 and 0.89 , indicating that the fitting index of the model meets the adaptation standard. The correlation coefficients of the four latent variables were concentrated between 0.6 and 0.9 , which met the significance level of 0.05 , indicating that there were higher-order influencing factors among the four latent variables, which needed to be analysed by a second-order model. Put the survey data into the newly constructed second-order model to analyse the value of each test index, and the results are shown in Table 1 and

Figure2.

Table 1 Model fitting results

\begin{tabular}{|c|c|c|c|c|c|}
\hline $\begin{array}{c}\text { Statistical } \\
\text { tests }\end{array}$ & $\chi^{2} / \mathrm{df}$ & RMSEA & GFI & AGFI & PGFI \\
\hline $\begin{array}{c}\text { Adapter } \\
\text { standard }\end{array}$ & $(1,3)$ & $<0.08$ & $>0.9$ & $>0.9$ & $>0.5$ \\
\hline $\begin{array}{c}\text { Inspection } \\
\text { standard }\end{array}$ & 1.817 & 0.071 & 0.923 & 0.907 & 0.603 \\
\hline
\end{tabular}




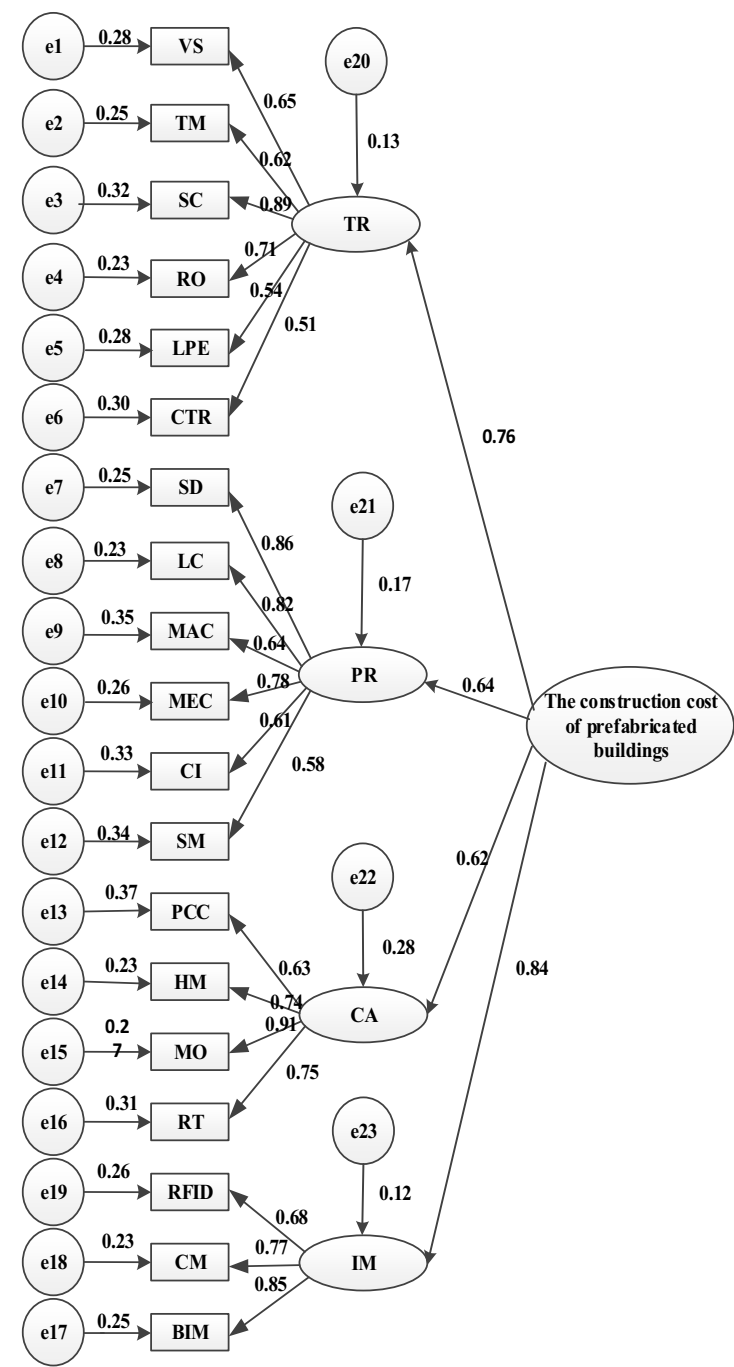

Fig.2. Normalized estimates of the structural model

\subsection{Analysis of model results}

According to the fitting results of the model in Table 1, it shows that all the hypotheses are valid. According to the results in Table 1 and Figure 2, the overall analysis is as follows:

(1) The impact of the production, transportation, assembly and application of information technology on the construction cost of prefabricated buildings is 0.64 , $0.76,0.62$ and 0.84 respectively, all of which are over 0.5 , indicating a significant impact. Therefore, the cost input in four dimensions should be controlled to reduce the construction cost of prefabricated buildings.

(2) The impact of the production cost of prefabricated parts on the transportation and on-site assembly cost is 0.62 and 0.74 respectively, showing a significant positive correlation, indicating that the production cost of prefabricated parts has a greater impact on the transportation and on-site assembly cost.

(3) The impact of information technology on the production, transportation and on-site assembly cost of prefabricated parts is $0.58,0.84$ and 0.89 , respectively, with significant impact. Therefore, the application of information technology in the transportation and assembly of prefabricated parts should be increased.
The results of each stage were analysed as follows:

(1) In the transportation stage of prefabricated parts, the location and route selection of prefabricated parts have a great impact on the transportation cost of prefabricated parts, which are 0.89 and 0.71 respectively. Therefore, the location selection and transportation route selection of prefabricated parts should be fully considered;

(2) In the production stage of prefabricated parts, the degree of standardization of prefabricated parts and the cost of mechanical use have a great impact on prefabricated parts, which are 0.86 and 0.76 respectively, indicating that prefabricated building standards are not uniform and the formulation of standards for prefabricated parts should be accelerated.

(3) In the field assembly stage of prefabricated parts, hoisting machinery and professional operators have a great impact on the cost, so controlling these two factors can reduce the cost of prefabricated parts assembly;

(4) The results of structural equation model show that information technology is very important. BIM, RFID and other technologies can design, optimize and monitor modules, so cutting-edge information technology can be fully applied to prefabricated buildings to reduce their costs.

\section{Conclusions}

In this paper, the structural equation model is used to verify the correlation between different dimensions of the three work spaces of the prefabricated components and the application of information technology. After analysis, the following conclusions are drawn:

(1) The construction cost analysis of prefabricated building based on structural equation model shows that the application of information technology and transportation of prefabricated parts have the greatest impact on the construction cost, while the production and assembly of prefabricated parts have little impact;

(2) The model system reflects the correlation between the stages of prefabricated components. The production cost of prefabricated components has a positive impact on transportation and assembly costs, while the application of information technology has a significant impact on the three stages.

(3) The standardized path coefficient of the model indicates that the location of the prefabricated parts plant, the optimization of the route, the standardization degree of prefabricated parts, the cost of mechanical use, hoisting machinery and professional operators are the key factors affecting the construction cost of prefabricated buildings.

Based on professional knowledge, the hypothesis of path influence was carried out in the study. However, the hypothesis model needs to be put into practice for verification, so it needs to be further investigated on site in future studies and the model should be improved by using big data.

\section{References}

1. Wang Defang, Zheng Shengqin, Guo Yanfeng. Research on influencing factors of construction 
Project cost control based on SEM [J]. Journal of Engineering Management, 2008, 32(1):53-58.

2. Chang Chunguang, Zhang Yu. Research on production cost Control of Prefabricated Building Components and Its Measures [J]. Journal of Shenyang Jian Zhu university (social science edition), 2016, 18(5):470-475.

3. He Jianguo. Research on distribution Cost Optimization of Prefabricated PC Components [J]. Urban Construction Theory Research (Electronic Version), 2017, (15):133-134.

4. Li Pingping. Research on distribution Cost Optimization of prefabricated PC Components [D]. $\mathrm{Xi}$ 'an: $\mathrm{Xi}$ 'an University of Architecture and Technology, 2016.

5. Barlow J, Childerhouse P, Gann D, et al. Choice and delivery in housebuilding: lessons from Japan for UK housebuilders [J]. Building Research \& Information, 2003, 31(2):134-145. 